\title{
TANGRA multidetector systems for investigation of neutron-nuclear reactions at the JINR Frank Laboratory of Neutron Physics
}

Ivan Ruskov ${ }^{1,2, *}$, Yury Kopach ${ }^{1}$, Vyacheslav Bystritsky ${ }^{1}$, Vadim Skoy ${ }^{1}$, Dimitar Grozdanov $^{1,2}$, Nikita Fedorov ${ }^{1}$, Tatyana Tretyakova ${ }^{3}$, Fuad Aliev ${ }^{4}$, Constantin Hramco ${ }^{5}$, Vyacheslav Slepnev ${ }^{1}$, Nikolay Zamyatin ${ }^{1}$, Aman Gandhi ${ }^{6}$, Dongming Wang ${ }^{7}$, Ajay Kumar ${ }^{6}$, Evgeni Zubarev ${ }^{8}$, Evgeny Bogolubov ${ }^{8}$, Yuri Barmakov ${ }^{8}$, and TANGRA collaboration

${ }^{1}$ Joint Institute for Nuclear Research (JINR), Joliot-Curie str., 6, 141980 Dubna, Moscow region, Russia

${ }^{2}$ Institute for Nuclear Research and Nuclear Energy (INRNE) of Bulgarian Academy of Sciences (BAS), Tzarigradsko Chaussee Blvd. 72, 1784 Sofia, Bulgaria

${ }^{3}$ Skobeltsyn Institute of Nuclear Physics (SINP), Lomonosov Moscow States University, Lenin Hills 1, 119991 Moscow, Russia

${ }^{4}$ Azerbaijan Institute of Geology and Geophysics of Azerbaijan National Academy of Sciences, H. Javid Av. 119, Baku AZ1143, Azerbaijan

${ }^{5}$ Institute of Chemistry of the Academy of Sciences of Republic of Moldova, Academic str. 3, MD-2028, Chisinau, Republic of Moldova

${ }^{6}$ Department of Physics, Banaras Hindu University, Varanasi - 221005, India

${ }^{7}$ School of Energy and Power Engineering, Xi'an Jiaotong University, Xianning West Road, Xi'an, Shaanxi, 710049, P.R. China

${ }^{8}$ All-Russia Research Institute of Automation (VNIIA), P.O. Box 918, 101000 Moscow, Russia

\begin{abstract}
In the framework of TANGRA-project at the Frank Laboratory of Neutron Physics of the Joint Institute for Nuclear research in Dubna (Russia), two experimental setups (Fig. 1) have been designed and tested for investigation of 14-MeV neutron-induced nuclear reactions on a number of important for nuclear science and engineering isotopes. As a source of 14-MeV "tagged" neutrons we are using the VNIIA ING-27 steady-state portable neutron generator with embedded in its vacuum tube 64-pixel charge-particle detector. The "Romashka" system is an array of up-to 24 hexagonal $\mathrm{NaI}(\mathrm{Tl})$-crystal scintillation probes, while the "Romasha" array consists of 18 cylindrical BGO-crystal detectors of neutrons and gamma-rays. In addition to these detectors there is a HPGe gamma-ray spectrometer and a number of Stilbene detectors that can be added for high-resolution gamma-ray spectrometry and neutron-gamma detection. The main characteristics of the neutron-induced nuclear reaction products can be investigated by commissioning the detectors in suitable for these experiments' geometries. Both setups can be used for doing basic and applied scientific research, because they permit simultaneously to measure the energy, angle and multiplicity distributions of gamma-rays and neutrons, produced in the competitive neutron-induced nuclear reactions (n, n' $\gamma),(\mathrm{n}, 2 \mathrm{n}),(\mathrm{n}, \mathrm{xn} \gamma)$ and $(n, f)$ in pure or complex substances.
\end{abstract}

\footnotetext{
*e-mail: ivan.n.ruskov@gmail.com;ivan@inrne.bas.bg
} 


\section{Introduction}

Our society and the modern technologies now and in future will need much more energy that in the past. To respond to this demand, different types of energy production sources are utilized or are in the stage of development (solar, wind, hydro, fission, fusion). Between them the most effective sources of clean energy still are the nuclear power plants. To solve the problems with the utilization of the burned fuel and radioactive wastes from Generation-IIII nuclear power reactors, Generation-IV fast neutron reactors are under development. The conceptual design, construction and more strict requirements for protecting the environmental need more precise data about the interaction of neutrons with atomic nuclei of nuclear fuel and the construction materials. The libraries with experimental and evaluated nuclear data, particularly on ${ }^{238} \mathrm{U}$, need to be enhanced with more precise and confident information about the characteristics (i.e. total and partial, differential, cross-sections) of different type of fast neutron induced reactions (elastic and nonelastic neutron scattering, fission, etc.), and of the products (mass-energy, multiplicity and angular distributions) from them [1,2]. The gammarays producing in the ( $\mathrm{n}, \mathrm{xn} \gamma)$ and (n, fission) are of a particular ifnterest from fundamental, practical and methodological points of view. They are helping to investigate the nuclear structure of the atomic nuclei as well as the neutron induced processes themselves, performing theoretical calculations, model simulations and/or experiments. Gamma-rays from (n, $\left.\mathrm{n}^{\prime} \gamma\right)$ and (n, f) reactions with fast neutrons scientifically contribute to the radiation heating and damage of the construction and radiation shielding materials. The results of calculations and simulations need to be validated in the experiments that are, as a rule, quite complicated, resource and time consuming. Because the gamma-rays are following the disintegration of any excited nucleus of the neutron irradiated samples and of the environmental materials, the experimental setups consist of neutron and/or gamma detectors shielded or not depends on the setup and the reaction(s) under investigation. In the case of fast neutron induced nuclear reaction many possibilities (channels) are possible (open), i.e. (n, xny), (n,p), (n, $\alpha),(\mathrm{n}, \mathrm{f})$, depends on the energy of the neutrons and the mass of the nuclei, and the use of multidetector systems is preferable, because in one measurement one can obtain, for example, the partial cross-section, angular and energy distributions of the reaction products. There are many and different types of multidetector systems for the investigation of the neutron induced reaction characteristics at neutron producing facilities all around the world.

At JINR FLNP in Dubna (Russia), in the frame of the international project TANGRA [3] we constructed 2 relatively low budget multidetector systems, namely, TANGRA-NaI(Tl) and TANGRA-BGO. TANGRA stands for TAgged Neutrons \& Gamma Rays. That means that we are using tagged neutrons for investigating the characteristics of the gamma rays by arrays of $\mathrm{NaI}(\mathrm{Tl})$ and BGO crystal based probes. As a source of tagged neutrons, we are using the portable neutron generator ING-27 of VNIIA (Moscow) [4].

\section{Experimental setups and their application}

TANGRA Setups are multidetector, multipurpose, multifunctional, mobile systems, to study the characteristics of the products from the nuclear reactions induced by $14-\mathrm{MeV}$ tagged neutrons [5-7]. TANGRA setups consist of a portable generator of "tagged" neutrons with energies of 14-MeV, ING-27, with or without an iron shield-collimator, 2D fast neutron beam profilometer, arrays of neutron-gamma detectors ("Romashka", "Romasha"), or single HPGe for high-resolution gamma-spectrometry and a computerized system for DAQ and analysis. 


\section{Portable Neutron Generator ING-27}

The ING-27 DT Neutron Generator, manufactured by VNIIA (Moscow, Russia), utilizes the ${ }^{3} \mathrm{H}(\mathrm{d}, \mathrm{n}){ }^{4} \mathrm{He}$ fusion reaction to produce $14-\mathrm{MeV}$ neutrons. A beam of deuterons is accelerated up to $\sim 120 \mathrm{keV}$ (in lab-system) and bombards a tritiated Ti-target in the generator assembly (TiT). The generator has a rated $14-\mathrm{MeV}$ neutron output of up to $10^{8} \mathrm{n} / \mathrm{s}$ in $4 \pi$ [4].
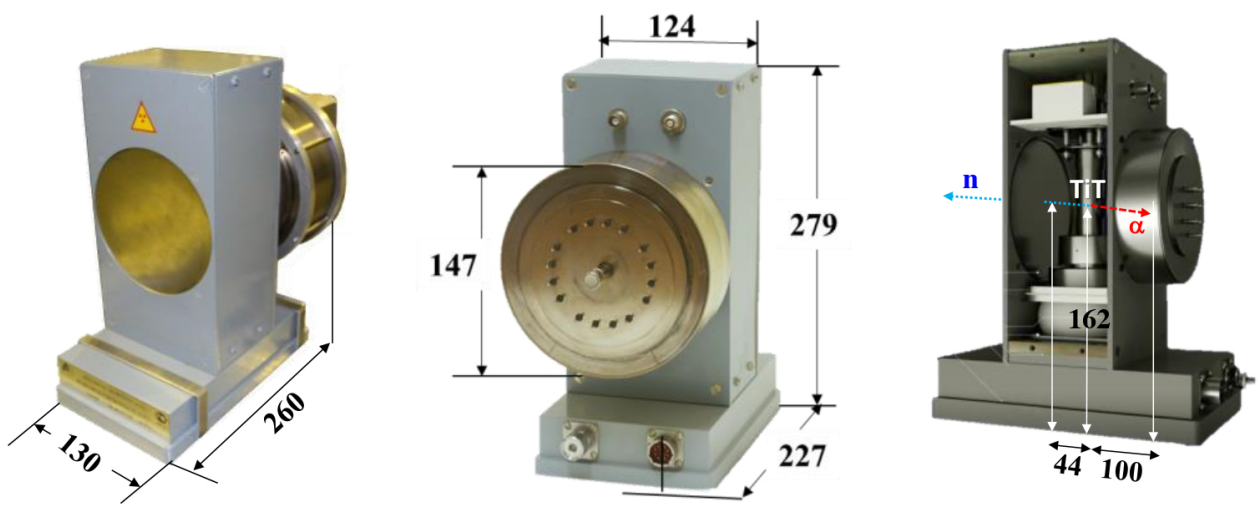

Figure 1. ING-27 dimensions $(\mathrm{mm})$ and the direction of irradiation of DT-reaction fragments.

The $\alpha$-particle detector incorporated into the ING-27 vacuum-chamber consists of $8 \mathrm{mu}$ tually perpendicular Si-strips on each side, mounted on a plastic frame and forming an $8 \times 8$ matrix of pixels with a size of $6 \times 6 \mathrm{~mm}$. The total sensitive area of the 64-element $\alpha$-detector is $55 \times 55 \mathrm{~mm}^{2}$. The pixelated position-sensitive alpha-detector is located $100 \mathrm{~mm}$ away from the centre of the TiT-target (Fig. 1, right). It permits to "tag" the neutron, i.e. to determine the direction cone of the neutron travel (x, y-resolution) and to give the start-time for measuring the neutron time-of-flight spectra ( $\mathrm{z}$, depth resolution). Its front-end electronics, which consists of 16 charge-sensitive pre-amplifiers, is mounted on the back side of the neutron generator.

All the 3 experimental TANGRA setups include an ING-27 as a source of tagged 14-MeV neutrons. For registering the gamma-rays produced in the interaction of the neutrons with the nuclei of the investigated sample and the environment we are using 2 arrays from $\mathrm{NaI}(\mathrm{Tl})$ and BGO crystal based detectors. "Romashka" multidetector system consists of up to 22 hexagonal NaI(Tl) crystal-based probes, "Romasha" BGO multidetector system consists of up to 18 cylindrical BGO crystal-based probes [5-7]. The $3^{\text {rd }}$ configuration at the moment has only one HPGe detector. "Romashka" $\mathrm{NaI}(\mathrm{Tl})$ radiation detectors can be arranged horizontally or vertically around the investigated sample at a desirable distance from it. The vertical configuration was chosen as having a bigger efficiency. "Romasha" BGO are arranged horizontally because of the crystal's geometry and dimensions. The main characteristics of the NaI(Tl), BGO and HPGe gamma-ray detectors are shown in Table 2. To measure the energy and angular distributions of gamma-rays form neutron induced nuclear reactions we are using $\mathrm{NaI}(\mathrm{Tl})$ and BGO detectors in configurations shown in Figure 2 (left and middle).

For obtaining the gamma-ray energy spectra with a high-resolution we are using a HPGe in a compact geometry, as shown in Fig. 2 (right). GEANT4 simulations of the experimental setups helped to obtain the optimal detector configurations, providing good efficiency for registration of gamma-rays and time-resolution. 
Table 1. ING-27 characteristics.

\begin{tabular}{lc}
\hline \multicolumn{2}{c}{ Neutron generator } \\
\hline TiT-to-front distance & $44.0 \pm 1.4 \mathrm{~mm}$ \\
TiT-to- $\alpha$-detector distance & $100 \pm 2 \mathrm{~mm}$ \\
Power supply voltage & $200 \pm 5 \mathrm{~V}$ \\
Max Power Supply Current & $300 \pm 30 \mathrm{mV}$ \\
Consumed Power & $<40 \mathrm{~W}$ \\
Weight: ING-27 & $7.5 \pm 0.5 \mathrm{~kg}$ \\
Weight: Power Supply and Operation Unit & $2.7 \pm 0.3 \mathrm{~kg}$ \\
n-tube life-time & $>800 \mathrm{~h}$ \\
$<$ ING-27 Duty time $>$ & $18 \mathrm{months}$ \\
\hline \multicolumn{1}{c}{ Double-side Si $\alpha$-particle detector } \\
\hline Number of pixels & $64(8 \times 8 \mathrm{strips})$ \\
Single pixel area & $6 \times 6 \mathrm{~mm} 2$ \\
Distance between strips & $0.5 \mathrm{~mm}$ \\
Voltage bias & $-250 \mathrm{~V} \mathrm{DC}$ \\
I_dark (250V DC, @ 20 $\left.{ }^{\circ} \mathrm{C}\right)$ & $<5 \mu \mathrm{A}$ \\
\hline
\end{tabular}

Table 2. The main characteristics of gamma-neutron detecting systems.

\begin{tabular}{lccc}
\hline Multi-detector array & NaI(Tl) "Romashka" & BGO "Romasha" & HPGe Ortec \\
\hline Number of detectors & 22 & 18 & 1 \\
Type & Crystal hexagonal rod & Crystal cylindrical rod & GMX 30-83-PL-S, \\
Dimensions & $(78 \times 90 \times 200 \mathrm{~mm})$ & $(\oslash 76 \times 65 \mathrm{~mm})$ & $(\oslash 57.5 \times 66.6 \mathrm{~mm})$ \\
Photo-multiplier tube & Hamamatsu R1306 & Hamamatsu R1307 & \\
$\gamma$-Energy-resolution & $\sim 7.2 \%(0.662 \mathrm{MeV})$ & $\sim 10.4 \%(0.662 \mathrm{MeV})$ & $\sim 3.4 \%(0.662 \mathrm{MeV})$ \\
$\gamma$-Energy-resolution & $\sim 3.6 \%(4.437 \mathrm{MeV})$ & $\sim 4.0 \%(4.437 \mathrm{MeV})$ & $\sim 0.3 \%(4.437 \mathrm{MeV})$ \\
$\gamma$-Time-resolution & $\sim 3.8 \mathrm{~ns}(4.437 \mathrm{MeV})$ & $\sim 4.1 \mathrm{~ns}(4.437 \mathrm{MeV})$ & $\sim 6.1 \mathrm{~ns}(4.437 \mathrm{MeV})$ \\
\hline
\end{tabular}
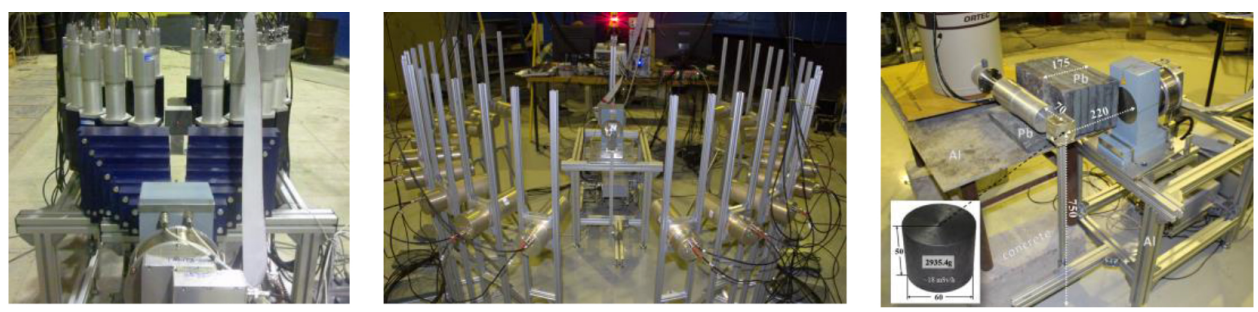

Figure 2. Experimental setups: TANGRA-NaI(Tl) (left), TABGRA-BGO (middle), TANGRA-HPGe (right).

\section{Tagged fast neutron beam profilometer}

To measure the tagged neutron beam profiles, we are using a 2D-detector of fast neutrons, made of 4 double-side stripped position-sensitive Si-detectors [8]. It is shown in Fig. 3 together with its main characteristics.

At the moment each 8 strips are grouped together, forming a $8 \times 8$-matrix with pixel size of $\sim 1.5 \times 1.5 \mathrm{~cm}$.

The setup for measuring the tagged neutron beam profiles is shown in Fig. 6. 


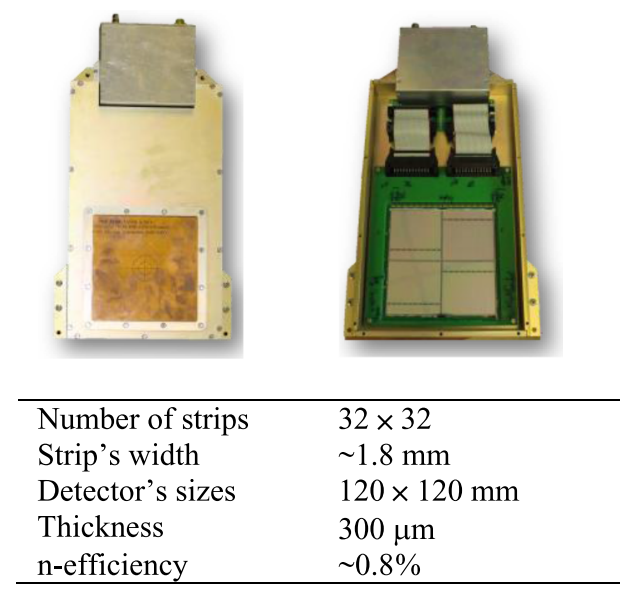

Figure 3. Fast neutron profilometer.

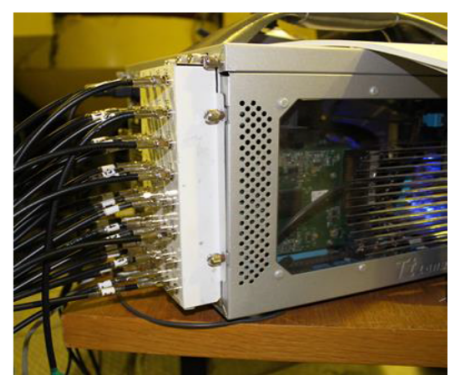

32-channel $100 \mathrm{MHz}, 14$ bit digitizer, in the form of 2 PCI-E cards; $10^{5}$ events/s for each input channel.

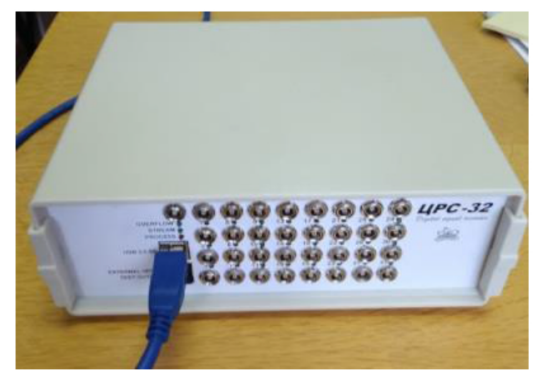

32-channel, $200 \mathrm{MHz}, 11$ bit digitizer, USB-3 connection; $\sim 10^{5}$ events/s for each input channel. This DAQ can work also with the HPGe detector.

Figure 4. AFI ADCM (left) and Digital Signal Recorder DSR-32 (right).

The neutron registration is done by detecting the charged particles (silicon recoil nuclei, protons, deuterons and alpha particles), formed as a result of the following neutron induced nuclear reactions: $\left.{ }^{28} \mathrm{Si}\left(\mathrm{n}, \mathrm{n}^{\prime}\right){ }^{28} \mathrm{Si},{ }^{28} \mathrm{Si}(\mathrm{n}, \alpha)\right)^{25} \mathrm{Mg},{ }^{28} \mathrm{Si}(\mathrm{n}, \mathrm{p}){ }^{28} \mathrm{Al}$ and ${ }^{28} \mathrm{Si}(\mathrm{n}, \mathrm{d})^{27} \mathrm{Al}$, during the interaction of fast neutrons with the substance of the profilometer (silicon).

\section{Data acquisition systems}

For collecting the amplitude signals from pixelized $\alpha$-sensor, $\gamma$-detector systems and $\mathrm{n}$ profilometer, we are using 2 computerized data acquisition 32-channel ADC systems: AFI Electronics ADCM and Alpatov's Digital Signal Recorder DSR-32, shown in Fig. 4.

The digitized amplitude signals (waveforms) are stored in list-mode (one-by-one) on the computer hard-disk for further off-line analysis by user created $\mathrm{C}++$ programs and CERN ROOT scripts. 


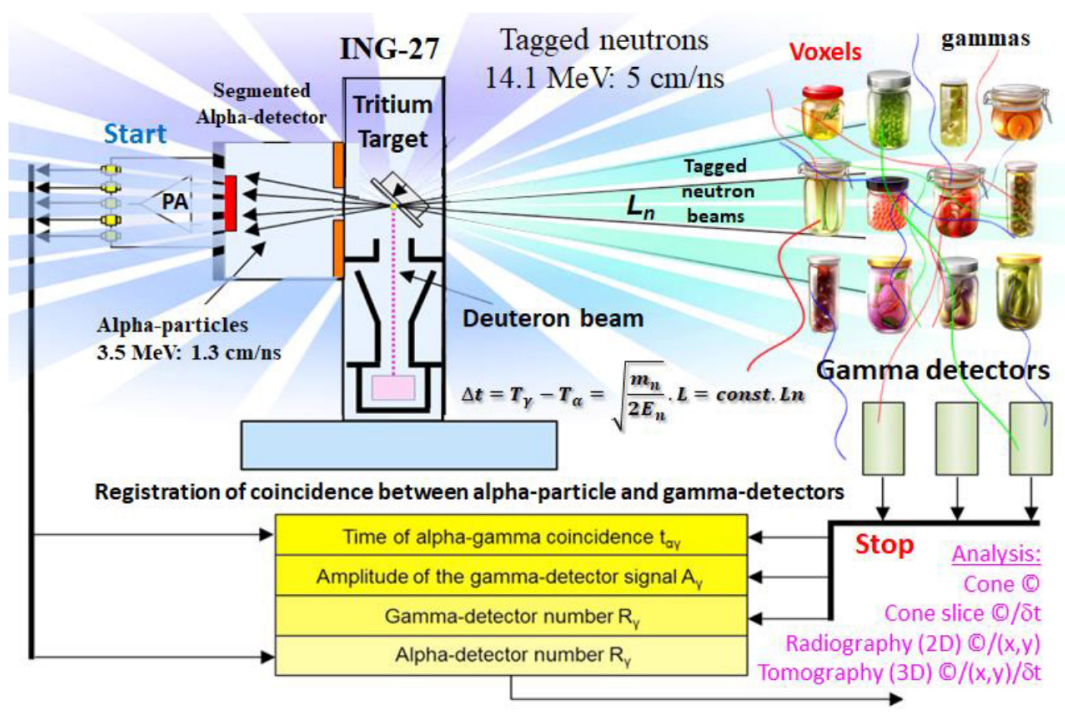

Figure 5. The scheme for investigating nuclear reactions by $14-\mathrm{MeV}$ neutrons, using time-correlated associated $\alpha$-particle technique.
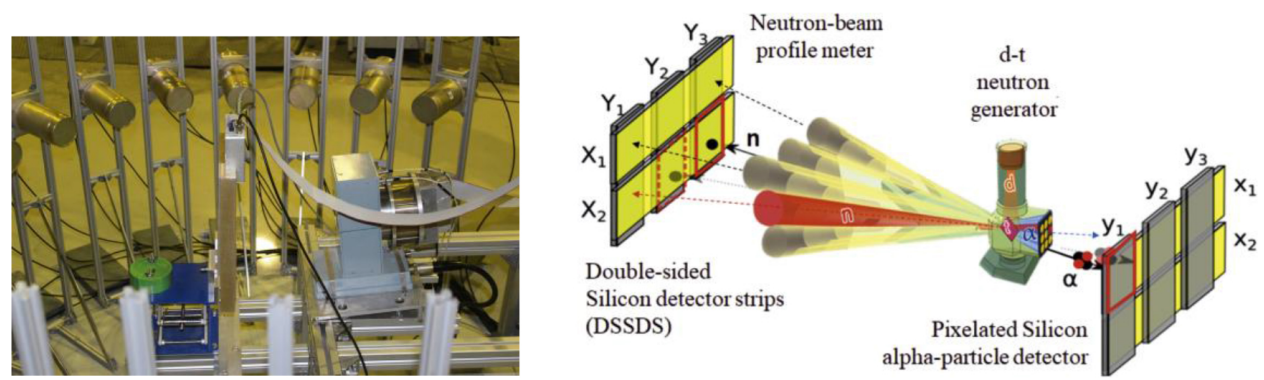

Figure 6. The photo of the setup (left) and the scheme for measuring the tagged neutron beam profiles (right).

\section{Application of the tagged neutron method}

For measuring the 14-MeV tagged neutron beam profiles and investigation the main characteristics of the neutron induced reactions we applied the Tagged Neutron Method (TNM), also known as Time-correlated Associated Particle (Imaging) Technique (Fig. 5).

The $14-\mathrm{MeV}$ neutron is tagged in time and direction by detecting the associated $\alpha$ particle, emitted in opposite direction (in CMS). The experimental setup for determination of the neutron flux in the ING-27 tagged neutron beams and their profiles with the n-profilometer by TNM is shown in Fig. 6 .

To know the neutron flux and its profiles with a high precision is very important for absolute determination of the neutron induced reaction cross sections. The number of registered $\alpha$-particles from DT-fusion reaction can be used as a monitor of neutrons irradiated from the TiT neutron producing target. The precision of monitoring depends on the detecting efficiency of the $\alpha$-sensor, as well as from the number of lost (absorbed, scattered) $\alpha$-particles prior to reach its sensitive area. We are using the n-profilometer for initial correct positioning 

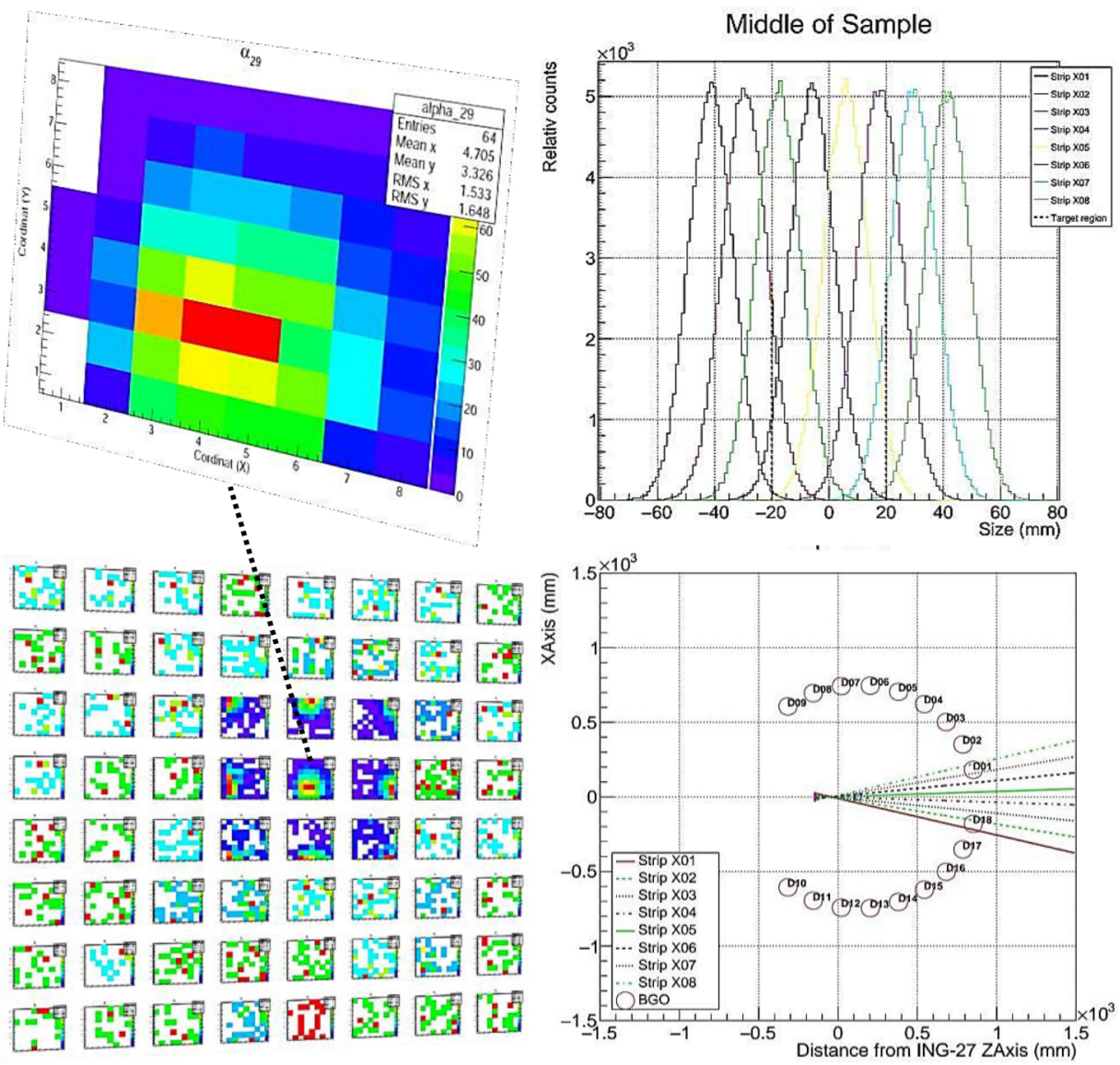

Figure 7. ING-27: 64 tagged neutron beams (down left), 2D-plot of one beam (up left), 8 tagged beams profiles (top right), azimuthal projection of 8 tagged beams cones (down right).

the target under investigation, to have as much as possible tagged beams hitting it, as well as detectors around it. An example of 2d-mapping of ING-27 tagged neutron beams, one of their profiles and the scheme of the experimental setup geometry with the beams' azimuthal projections are shown in Fig. 7.

Knowing the time "stamps" and the amplitudes of the signals it is possible not only to determine the neutron flux irradiating a certain voxel of the investigated object, but also to determine its location and elemental (isotope) composition. Situating the gamma (neutron) detectors around the sample in a way to avoid the multiple scattering effect, it is possible to measure the angular distribution of gamma-rays (and/or neutrons) and to obtain the angular correlations between them also, in addition to their energy spectra.

The associated particle technique for fast neutron interrogation of bulk samples is widely used for detection of explosives, buried land mines, chemical weapons, unexploded ordnance, drugs detection, in-vivo body composition, as well as of special nuclear materials (SNM).

By means of 2 multidetector systems, we made a feasibility study for measuring the angular distributions of gamma-rays from the inelastic scattering of $14.1-\mathrm{MeV}$ neutrons on the nuclei of a number of light and middle weight isotopes, important for nuclear sciences, for which these data are not available or are known with a nonsufficient accuracy. 


\section{Test measurements with a bulk sample from ${ }^{\text {nat }} \mathrm{U}$}

To investigate the sensitivity of our TANGRA BGO and HPGe setups (Fig. 2, centre and right photos) for measuring the energy, anisotropy and multiplicity distributions of gammarays and the correlation of gamma-rays with the neutrons from the $(\mathrm{n}, \mathrm{xn} \gamma),(\mathrm{n}, \mathrm{f}),(\mathrm{n}, \mathrm{nf})$ reactions of 14-MeV neutrons on nuclei of some SNM, we made a short-term test with a bulk metal sample from natural uranium $\left({ }^{\text {nat }} U\right)$.

The relative isotopic abundance of the sample is shown in Table 3.

Table 3. Relative Isotopic Abundance of the sample from ${ }^{\text {nat }} \mathrm{U}$.

\begin{tabular}{rrr}
\hline Isotope & By weight & By activity \\
\hline U-238 & $99.282 \%$ & $48.8 \%$ \\
U-235 & $0.712 \%$ & $2.4 \%$ \\
U-234 & $0.006 \%$ & $48.8 \%$ \\
\hline
\end{tabular}

Many factors may interfere with the prompt $\gamma$-ray emission from the inelastic scattering $(\mathrm{n}, \mathrm{n}, \gamma)$ and fission (n,f) reactions, most of which are listed here: (1) delayed $\gamma$-rays emitted from the natU-sample; (2) prompt $\gamma$-rays from the T-target, ING-27 construction materials; (3) $\gamma$-rays from the detector and its surrounding material irradiated by scattered neutrons; (4) prompt $\gamma$-rays from the detector generated by penetrating neutrons; (5) prompt $\gamma$-rays from the Al-support of ING-27 and BGO-detectors; (6) spontaneous fission $\gamma$-rays from the sample.

The experimental data are in the process of analyzing and the results from it will be published elsewhere in a separate paper. Here, to highlight the problems one can have when is doing this type of measurements with SNM and to show the importance of using tagged neutrons and the TCAPM, we show only the energy spectra, obtained with TANGRA-HPGe setup (Fig. 2, right) and a rod-sample from ${ }^{\text {nat }} \mathrm{U}$ in the $\gamma$-ray energy interval from $60 \mathrm{keV}$ up to $2 \mathrm{MeV}$. The measurements that were done are also indicated in Fig. 8. There are gamma-peaks from the ${ }^{27} \mathrm{Al}(\mathrm{n}, \mathrm{n} / \gamma)$ and peaks from the natural $\gamma$-radioactivity of the sample. The best way to minimize the influence of these parasitic sources of gammas is to apply the TCAPM, by which one can extract the gamma-ray energy spectra in different time-windows of the collected time-spectra, making the influence of the expected radiation background on the final results negligible.

\section{Summary and outlook}

In the framework of TANGRA project at JINR-FLNP were constructed 3 experimental setups for investigating the multiplicity, energy and angular distribution of gamma-rays and neutrons from induced by tagged $14-\mathrm{MeV}$ neutrons $(\mathrm{n}, 2 \mathrm{n}),(\mathrm{n}, \mathrm{xn} \gamma)$ and $(\mathrm{n}, \mathrm{f})$ nuclear reactions. The experimental setups, consisted of ING-27 portable neutron generator and $\mathrm{NaI}(\mathrm{Tl})$, BGO, HPGe detectors, were successfully tested and used for measuring the angular distributions of gamma-rays from the inelastic scattering of $14-\mathrm{MeV}$ neutrons with a number of materials used or supposed to be used as construction materials of today and future nuclear fission and fusion reactors. The further research activities are connected also with determination of neutron and gamma-ray emission from (n, n $\gamma$ ) and (n, f) reactions of 14-MeV tagged neutrons with ${ }^{238} \mathrm{U}$ and other fertile and fissile heavy isotopes. 

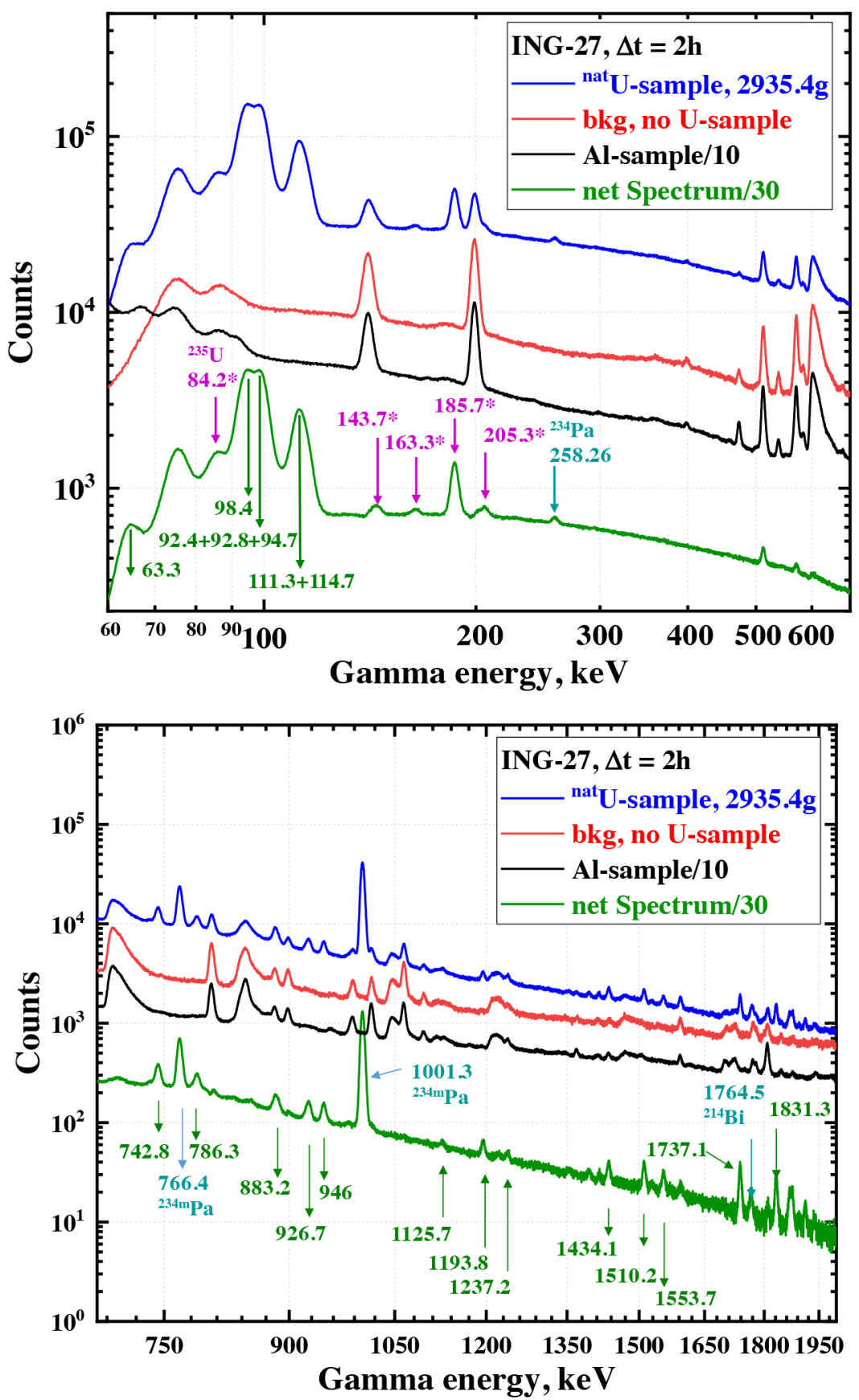

Figure 8. Energy spectra of the gamma-rays from ${ }^{\text {nat }} \mathrm{U}$-sample.

\section{References}

[1] R. Capote, A. Trkov, M. Sin, M. Herman, A. Daskalakis, and Y. Danon, Physics of Neutron Interactions with ${ }^{238} \mathrm{U}$ : New Developments and Challenges, Nuclear Data Sheets 118 (2014) 26-31, doi:10.1016/j.nds.2014.04.003 
[2] Roberto Capote and Andrej Trkov, Critical review of CIELO evaluations of $\mathrm{n}+$ ${ }^{235} \mathrm{U},{ }^{238} \mathrm{U}$ using differential experiments, EPJ Nuclear Sci. Technol. 4 (2018) 27, doi:10.1051/epjn/2018029

[3] Project TANGRA: "Design and development of the tagged neutron method for nuclear reaction studies and determination of the elemental structure of substances", JINR, Dubna, Russian Federation, http://flnph.jinr.ru/en/facilities/tangra-project

[4] E.P. Bogolyubov, A.V. Gavryuchenkov, M.D. Karetnikov, D.I. Yurkov, V.I. Ryzhkov, V.I. Zverev, Neutron generators and DAQ systems for tagged neutron technology, Proceedings of the XXVI International Symposium on Nucl. Electronics \& Computing (NEC'2017), Becici, Budva, Montenegro, September 25-29, (2017) 176-181, http: //ceur-ws.org/Vol-2023/176-181-paper-27.pdf, http://test.vniia.ru/eng/ng/element.html

[5] I.N. Ruskov, Yu.N. Kopatch, V.M. Bystritsky, V.R. Skoy, V.N. Shvetsov, F.-J. Hambsch, S. Oberstedt, R. Capote Noy, P.V. Sedyshev, D.N. Grozdanov, I.Zh. Ivanov, V.Yu. Aleksakhin, E.P. Bogolubov, Yu.N. Barmakov, S.V. Khabarov, A.V. Krasnoperov, A.R. Krylov, J. Obhodaš, L.B. Pikelner, V.L. Rapatskiy, A.V. Rogachev, Yu.N. Rogov, V.I. Ryzhkov, A.B. Sadovsky, R.A. Salmin, M.G. Sapozhnikov, V.M. Slepnev, D. Sudac, O.G. Tarasov, V. Valkovic, D.I. Yurkov, N.I. Zamyatin, Sh.S. Zeynalov, A.O. Zontikov, E.V. Zubarev. TANGRA-Setup for the Investigation of Nuclear Fission Induced by 14.1 MeV Neutrons, Physics Procedia 64 (2015) 163-170, doi:10.1016/j.phpro.2015.04.022

[6] I.N. Ruskov, Yu.N. Kopatch, V.M. Bystritsky, V.R. Skoy, V.N. Shvetsov, F.-J. Hambsch, S. Oberstedt, R. Capote Noy, and TANGRA collaboration, TANGRA-Setup for the Investigation of Nuclear Fission Induced by 14.1 MeV Neutrons, Scientific Workshop on Nuclear Fission dynamics and the Emission of Prompt Neutrons and Gamma Rays, THEORY-3, Physics Procedia 64 (2015) 163-170, doi:10.1016/j.phpro.2015.04.022

[7] Ivan Ruskov, Yury Kopatch, Vyacheslav Bystritsky, Vadim Skoy, Valery Shvetsov, Franz-Josef Hambsch, Stephan Oberstedt, Roberto Capote Noy, Dimitar Grozdanov, and TANGRA collaboration, TANGRA - an experimental setup for basic and applied nuclear research by means of $14.1 \mathrm{MeV}$ neutrons, ND2016, EPJ Web of Conferences 146, 03024 (2017), doi:10.1051/epjconf/201714603024.

[8] Zamyatin N.I., Bystritsky V.M., Kopach Y.N., Aliev F., Grozdanov D.N., Fedorov N.A., Hramko C., Ruskov I.N., Skoy V.R., Sleplev V.M., Wang D., Zubarev E.V., Neutron beam profilometer on the base of double-sided silicon strip detectors. Nuclear Instruments and Methods in Physics Research A898 (2018) 46-52, doi:10.1016/j.nima.2018.04.031 